\title{
Deformation and crustal rigidity of rotating neutron stars
}

\author{
J. L. Zdunik ${ }^{1}$, M. Bejger ${ }^{2,1}$, and P. Haensel ${ }^{1}$ \\ 1 N. Copernicus Astronomical Center, Polish Academy of Sciences, Bartycka 18, 00-716 Warszawa, Poland \\ e-mail: [jlz; bejger; haensel] $a_{\text {camk .edu.pl }}$ \\ 2 LUTH, UMR 8102 du CNRS, Observatoire de Paris, 92195 Meudon Cedex, France
}

Received 13 May 2008 / Accepted 22 August 2008

\section{ABSTRACT}

\begin{abstract}
Aims. We calculate parameters $A$ and $B$ of the Baym-Pines model of the hydro-elastic equilibrium of rotating neutron stars. Parameter $A$ determines the energy increase of a non-rotating star due to a quadrupolar deformation of its shape. Parameter $B$ determines residual quadrupolar deformation due to the crustal shear strain in a neutron star that spun down to a non-rotating state. Methods. The calculations of $A$ are based on precise numerical 2D calculations for rotating neutron stars with the realistic equations of state (EOSs) of dense matter. An approximate, but quite precise, formula for $B$ is used, which allows us to separate the contribution of the crust from the dependence on the stellar mass $M$ and radius $R$. The elastic shear strain distribution within the crust is modeled following Cutler et al. (2003). Realistic EOSs of neutron star cores are used, some of them with high-density softening due to the appearance of hyperons or a phase transition to an exotic state.

Results. The values $A(M)$ and $B(M)$ were calculated for $0.2 M_{\odot}<M<0.9 M_{\max }$ (where $M_{\max }$ is the maximum allowable mass) for seven EOSs of neutron star core, combined with several crust models. A standard formula based on the incompressible fluid model is shown to severely underestimate the value of $A$. For $M<0.7 M_{\odot}$ the values of $A(M)$ are nearly EOS-independent and are given (within a few percent) by a universal formula $A=3.87\left(M / M_{\odot}\right)^{7 / 3} \times 10^{53} \mathrm{erg}$. We derive the scaling of $B$ with respect to $R$ and $M$, also valid for a thick crust. We show that $B$ for accreted crust strongly depends on pycnonuclear fusions at $\rho>10^{12} \mathrm{~g} \mathrm{~cm}^{-3}$.
\end{abstract}

Key words. dense matter - equation of state - stars: neutron

\section{Introduction}

The solid crust of neutron stars forms quickly after their birth. It plays an important rôle in neutron-star dynamics. Apart from pressure, the stresses in the crust are produced by an elastic shear strain; therefore, the crust can have a non-axial deformation of mass distribution, which allows a rotating neutron star to radiate gravitational waves. Moreover, the inner neutron-star crust, with a crystal lattice of nuclei immersed in neutron superfluid, can be a site of pinning the neutron vortices to nuclei. A massive breaking of this pinning after a maximal strain has been reached is thought to be responsible for triggering the pulsar glitches. Finally, the presence of the crust makes a free precession of a rotating neutron star possible.

The hydrostatics of the neutron star crust is more complicated than for an ideal fluid, because of the elastic strain that produces an anisotropic contribution to the stress tensor. To find the mechanical equilibria of a crust with non-vanishing shear strain, one has to solve partial differential equations of the hydroelastic equilibrium, with appropriate boundary conditions on the stellar surface and on the crust - liquid core boundary. A simple "one-parameter model" of a neutron-star structure with crustal elastic strain and its application to pulsar glitches was given by Baym \& Pines (1971). The Baym-Pines model was reformulated in the framework of General Relativity by Carter \& Quintana (1975). A neutron-star precession model was developed by Pines \& Shaham (1972a,b), who used methods originally developed to describe rotation of the Earth (Munk \& McDonald 1960, and references therein). In these models, the neutron star is treated as a solid shell (crust) enveloping a liquid core. More recently, updated review of models of precessing neutron stars was presented by Jones \& Andersson (2001). The crustal rigidity, resulting from elastic strain in the deformed crust of neutron stars, was recently reexamined, using realistic dense matter models by Cutler et al. (2003).

The change in the stellar energy due to star's quadrupolar deformation is determined by the parameter $A$ of Baym \& Pines (1971). In the present paper we calculate $A$ versus stellar mass $M$, using quadratic expansions of exact quantities obtained in the precise $2 \mathrm{D}$ simulations. To this aim, we have developed a systematic procedure enabling us to calculate a quadratic response of neutron star structure to a rigid rotation. Using a set of EOSs, we study the EOSs dependence of $A(M)$. Then we calculate the parameter $B$ measuring the elastic strain contribution to the hydro-elastic equilibrium of a rotating star with crust. In doing this, we avoid solving the complete hydro-elastic equilibrium equations, by relying on results obtained by Cutler et al. (2003), and adapting them to different EOSs of the crust and core. We then study the scaling of $B$ with the mass and the radius of neutron star and investigate the differences between the ground-state and accreted crusts.

The plan of the paper is as follows. The model of Baym and Pines is briefly described in Sect. 2, where the parameters $A$ and $B$ are defined. Calculation of $A$, based on the $2 \mathrm{D}$ simulations for rigidly rotating neutron stars with realistic EOSs, is described in Sect. 3. The shear modulus of the crusts is briefly reviewed in Sect. 4, where we also discuss the differences between the ground-state and accreted crusts. An approximation suitable for calculating the crustal rigidity parameter, valid also beyond the limit of a thin crust, is proposed in Sect. 5. Existing calculations of $B$, and our results for this quantity, are described in Sects. 67. Scaling of $B$ with $M$ and $R$ is briefly discussed in Sect. 8 . 
Numerical results for crustal rigidity versus stellar mass are presented in Sect. 9. Discussion of our results and conclusions are presented in Sect. 10.

\section{The Baym-Pines model}

We restrict ourselves to neutron star stars with an ellipsoidal (quadrupole) deformation of an axially symmetric crust. The rest mass of the star is fixed and equal to $M_{\mathrm{b}}$. The stress tensor of dense matter is $\sigma_{i j}=\sigma_{i j}^{\mathrm{liq}}+\sigma_{i j}^{\mathrm{sh}}$, where the dominant, ideal liquid term $\sigma_{i j}^{\text {liq }}=-P \delta_{i j}, P$ is the pressure, and an elastic shear stress component $\sigma_{i j}^{\text {sh }}$ (see Sect. 5) exists only within the solid crust. Correspondingly, the energy of a configuration is $E=E^{\mathrm{liq}}+E^{\mathrm{sh}}$.

Non-sphericity of an axially symmetric rotating configuration results in a moment of inertia tensor $\breve{I}_{i j}=\operatorname{diag}\left(\breve{I}_{1}, \breve{I}_{1}, \breve{I}_{3}\right)$. The quadrupolar deformation is fully described by the oblateness parameter

$\epsilon \equiv \frac{I_{3}-I_{0}}{I_{0}}$

and diagonal elements of $I_{i j}$ are

$I_{1}=I_{2}=I_{0}(1-\epsilon / 2), \quad I_{3}=I_{0}(1+\epsilon)$,

so that the trace of $I_{i j}$ (invariant with respect to rotations of the coordinate system) is $3 I_{0}$.

Our definition of oblateness parameter requires a comment. It is the same as used by Cutler et al. (2003); however, it differs from that of Baym \& Pines (1971), who defined it in terms of the crust moment of inertia, $\epsilon_{\mathrm{BP}}=\left(I_{\mathrm{c} 3}-I_{\mathrm{c} 0}\right) / I_{\mathrm{c} 0}$. For stars made of incompressible fluid, $\epsilon=\epsilon_{\mathrm{BP}}$; however, for realistic stars, made of compressible matter, $\epsilon>\epsilon_{\mathrm{BP}}$.

Two reference configurations are defined as follows.

$C_{0}$-non-rotating and relaxed. It is spherical, with $E^{\mathrm{sh}}\left(C_{0}\right)=$ 0 (no shear stress, completely relaxed) and has energy $E_{0}$. Its moment of inertia tensor is $I_{i j}^{0}=I_{0} \delta_{i j}$.

$\breve{C}$ - rotating and relaxed. It is rigidly rotating, at rotation frequency $\breve{\Omega}=2 \pi /($ rotation period). This configuration is also completely relaxed, so that $E^{\mathrm{sh}}(\breve{C})=0$.

An example of a reference configuration $\breve{C}$ is a newly born neutron star that is already rigidly rotating but still completely liquid. Then the star spins down, and its ellipticity decreases to $\epsilon<\breve{\epsilon}$. However, the crust solidifies quickly, and elastic strain accumulates within the crust, which is opposing the decrease in $\epsilon$.

Let us consider a rotating configuration $C$, with rotation frequency $\Omega$, and born as $\breve{C}$. Assume that there is a continuous spin-down transformation $\breve{C} \longrightarrow C$ (no crust breaking). Then, a "residual oblateness", which adds to that resulting from rotation, is (Baym \& Pines 1971; Carter \& Quintana 1975; Cutler et al. 2003)

$\epsilon_{\mathrm{r}}=b \breve{\epsilon}$.

Here, $b$ is a "rigidity parameter" (called so by Jones \& Andersson 2001), which depends on the elastic properties of the crust, on the EOS of neutron star matter, and on the mass $M$ of the reference configuration $C_{0}$. The name "residual oblateness" stems from this being the ellipticity that remains after a complete spin down to $\Omega=0$.

Within the Baym-Pines model, the value of $b$ can be calculated by considering the limit of a non-rotating star. The quadratic approximation to the stellar energy is then

$E=E_{0}+A \epsilon^{2}+B(\epsilon-\breve{\epsilon})^{2}$, where parameter $A$ is calculated from the quadratic response of a non-rotating neutron star energy to a quadrupole deformation. The last term on the righthand-side is $E^{\text {sh }}$, verifying $E^{\mathrm{sh}}(\breve{C})=0$. The equilibrium of a non-rotating star is reached at minimum $E$, which implies

$\epsilon_{\mathrm{r}}=\frac{B}{A+B} \breve{\epsilon} \Longrightarrow b=\frac{B}{A+B}$.

In their calculation of $b$, Cutler et al. (2003) rely directly on the difference $\Delta I=I_{3}-I_{0}=I_{0} \epsilon$, calculated in two cases. The value $\Delta I_{\Omega}=I_{0} \breve{\epsilon}$ is obtained for a fully relaxed configuration rotating at $\Omega$, while the residual $\Delta I_{\mathrm{d}}=I_{0} \epsilon_{\mathrm{r}}$ results from a continuous spin-down from $\Omega$ to zero. Within the quadratic approximation in $\Omega$, one gets $\Delta I_{\mathrm{d}} / \Delta I_{\Omega}=b=\epsilon_{\mathrm{r}} / \breve{\epsilon}$.

Early calculations for a $1.4 M_{\odot}$ neutron star have given $A \sim 10^{53}$ erg and $B \sim 10^{48}$ erg (Pandharipande et al. 1976), so that $b \simeq B / A \simeq 10^{-5}$. Recent calculations for a $1.4 M_{\odot}$ neutron star with realistic EOSs of neutron star crust and core yields a much lower value $b=(1.7-2.5) \times 10^{-7}$ (Cutler et al. 2003). Dependence of $A$ and $B$ on the stellar mass was studied by Carlini \& Treves (1989). As we will see, the approximations used by Carlini \& Treves (1989) were rather unrealistic.

\section{Rotational deformation of a relaxed configuration}

We intend to calculate parameter $A$ as a function of the gravitational mass of the non-rotating configuration of the same baryon mass, $M_{\mathrm{b}}$. Consider configuration rotating rigidly at angular frequency $\Omega$, as measured by a distant observer. In what follows, we use the results of exact $2 \mathrm{D}$ calculations. Einstein equations for stationary axi-symmetric space-time are solved using the rotstar code, a part of the LORENE public-domain $\mathrm{C}++$ scientific library based on spectral methods of solution of partial differential equations (see http://www. lorene. obspm. fr/). The stellar angular momentum is $J$ and mass-energy is $M c^{2}$. In what follows, in the spirit of the Baym-Pines model, we restrict ourselves to a slow rotation limit; i.e., we keep only lowest powers of $\Omega$. To extract relevant coefficients, we have chosen a non-dimensional expansion parameter $\bar{\Omega}$ equal to the angular frequency $\Omega$ divided by the angular frequency for a star rotating at $10 \mathrm{~ms}$ period, $\bar{\Omega}=\Omega / \Omega_{10 \mathrm{~ms}}=\Omega /\left(2 \pi \cdot 100 \mathrm{~s}^{-1}\right)$.

\subsection{Coefficients $\alpha$ of the star's response to rotation}

The non-sphericity of the mass-distribution is measured by ellipticity $\breve{\epsilon}$. We calculate and expand $I_{3}$ using

$I_{3}=\frac{J}{\Omega}=I_{0}\left(1+\alpha_{I} \bar{\Omega}^{2}\right)$,

which yields

$\breve{\epsilon}=\alpha_{I} \bar{\Omega}^{2}$.

Simultaneously, we can parametrize the geometrical oblateness of the shape of rotating star by

$1-r_{\mathrm{pol}} / r_{\mathrm{eq}}=\alpha_{\mathrm{s}} \bar{\Omega}^{2}$,

where $r_{\mathrm{pol}}\left(r_{\mathrm{eq}}\right)$ is the polar (equatorial) radial coordinate of rotating star. Let us now consider the decomposition of the total mass-energy $M c^{2}$ into the static part and kinetic energy. The kinetic energy is defined by analogy to the Newtonian theory (Friedman et al. 1986)

$T=\frac{1}{2} J \Omega=\frac{1}{2} I \Omega^{2}$. 

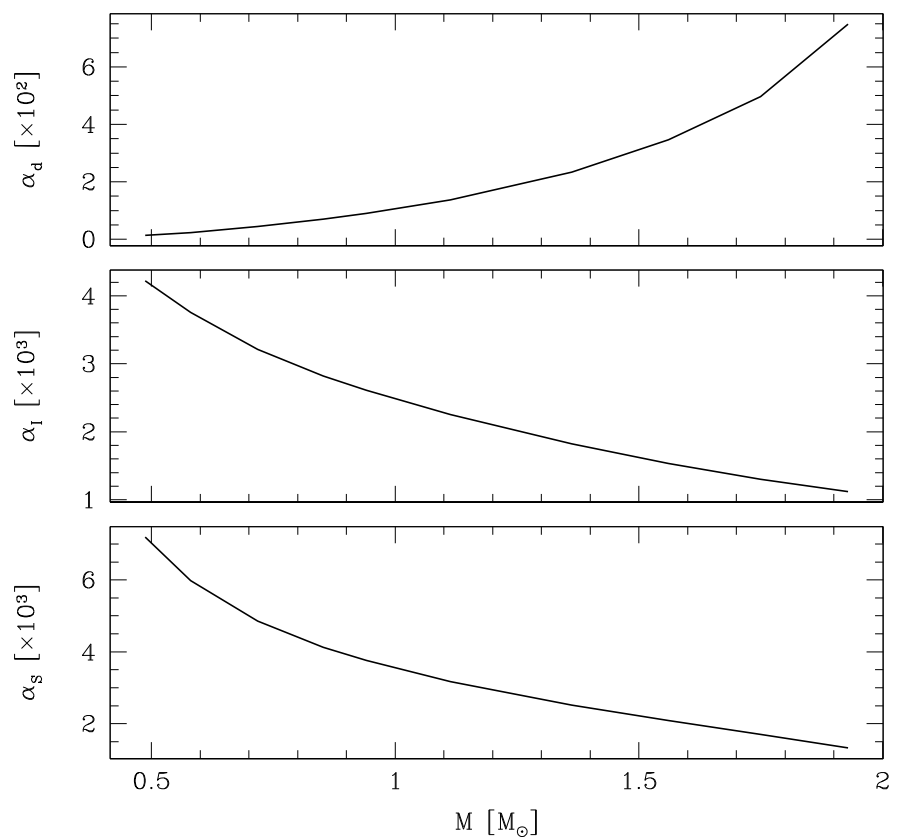

Fig. 1. Parameters $\alpha_{\mathrm{s}}, \alpha_{I}$, and $\alpha_{\mathrm{d}}$ versus stellar mass $M$, calculated for the DH EOS (Table 1). For further explanations see the text.

Therefore,

$M c^{2}=M^{(0)} c^{2}+E_{\mathrm{def}}+T$,

where $M^{(0)} c^{2}$ is mass-energy of non-rotating configuration, and $E_{\mathrm{def}}$ is the increase in intrinsic energy of the star (i.e., calculated in the star's reference system), as compared to the nonrotating (static) case. To the lowest order, the latter quantity can be parametrized as

$E_{\mathrm{def}}=\alpha_{\mathrm{d}}\left(1-r_{\mathrm{pol}} / r_{\mathrm{eq}}\right)^{2} M_{\odot} c^{2}$.

The parameters $\alpha$ were calculated, using exact 2D models of rotating neutron stars, for several realistic EOSs of neutron star cores. These EOSs are listed in Table 1. As the EOS of neutron star crust, we used the model of Douchin \& Haensel (2001) (hereafter referred to as DH). Examples of the dependence of the $\alpha$ parameters on the gravitational mass of neutron star are shown in Figs. 1 (DH EOS) and 2 (BGN1H1 EOS). The DH EOS assumes that the matter is composed exclusively of nucleons and leptons, and it leads to a monotonous behavior of all $\alpha$-s. Namely, $\alpha_{I}$ and $\alpha_{\mathrm{s}}$ decrease with increasing $M$. Such behavior is quite natural, because $\alpha_{\mathrm{s}}$ and $\alpha_{I}$ measure the response of the shape and mass distribution to a rotation at a fixed $\Omega$. This response (polar flattening, anisotropy of the moment of inertia tensor) weakens with increasing mass, because gravitational pull, which tends to concentrate the matter towards the center, becomes stronger. On the contrary, the increase in energy, relative to the spherical shape, due to a given ellipticity of the star, is measured by $\alpha_{\mathrm{d}}$. This energy rapidly increases with star mass: gravitational binding of the star rises with $M$, and the star becomes stiffer to polar flattening.

There are some bounds on $M$ resulting from the high-precision constraint imposed on the 2D simulations. Calculations of $\alpha$-s had to be stopped at $M \simeq 0.9 M_{\max }$, because for $M \approx M_{\max }$, the closeness of relativistic instability makes precise 2D calculation of linear response to $\bar{\Omega}^{2}$ very difficult. On the other hand, $M$ could not be too low, because for
Table 1. Equations of state of neutron star core.

\begin{tabular}{ccc}
\hline \hline EOS & Model & Reference \\
\hline BPAL12 & N energy density functional & $\mathrm{a}$ \\
FPS & N energy density functional & $\mathrm{b}$ \\
GN3 & N relativistic mean field & $\mathrm{c}$ \\
DH & N energy density functional & $\mathrm{d}$ \\
APR & N variational theory & $\mathrm{e}$ \\
BGN1H1 & NH, energy density functional & $\mathrm{f}$ \\
GNHQm2 & $\mathrm{NH}+$ mixed baryon-quark state & $\mathrm{g}$ \\
\hline
\end{tabular}

Note: $\mathrm{N}$ - nucleons and leptons. $\mathrm{NH}$ - hyperons, nucleons, and leptons. References for the EOS: a - Bombaci (1995); b - Pandharipande \& Ravenhall (1989); c - Glendenning (1985); d - Douchin \& Haensel (2001); e - A18 $\delta+$ UIX $^{*}$ model of Akmal et al. (1998); f - Balberg \& Gal (1997); g - Glendenning (2000).
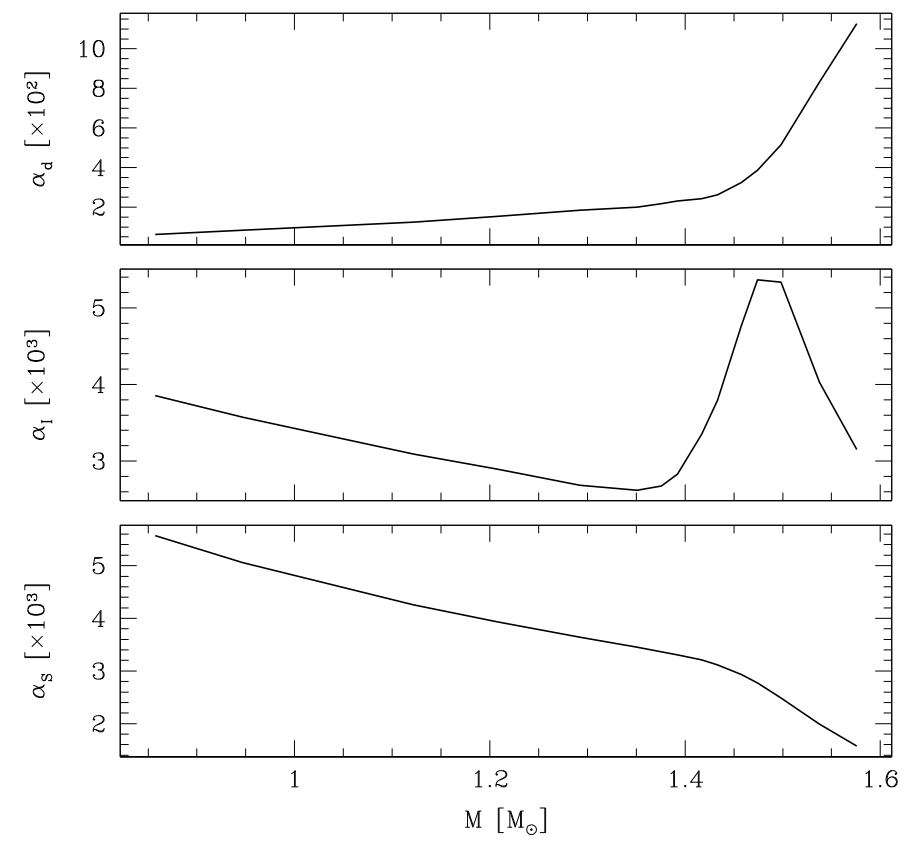

Fig. 2. Same as Fig. 1 but calculated for the BGN1H1 EOS (Table 1).

low $M$ the rotating star becomes very "soft" to rotational deformation, which is reflected in very high values of $\alpha_{\mathrm{s}}$ and $\alpha_{I}$. All in all, very precise calculation of $\alpha$-s could be done only for $0.2 M_{\odot} \lesssim M \lesssim 0.9 M_{\max }{ }^{1}$.

The BGN1H1 EOS takes the high-density softening due to the appearance of hyperons into account. However, for $M \lesssim$ $1.35 M_{\odot}$ stellar core does not contain hyperons, and the $\alpha(M)$ dependence is monotonous, similar to the DH EOS. For $M>$ $1.35 M_{\odot}$ hyperons soften the neutron star core, resulting in a specific $M(R)$ dependence. Namely, $M$ increases very weakly with a decreasing $R$ (flat $M(R)$ curve). In contrast, one notes a strong increase in the binding energy of the star, and a decrease in the moment of inertia with increasing $M$, Fig. 3 . The effects of the hyperon softening on $\alpha(M)$-s are therefore the following. The function $\alpha_{\mathrm{s}}\left(\alpha_{\mathrm{d}}\right)$ decreases (increases) more steeply with $M$, because the star becomes more compact and its binding increases rapidly with $M$. The most characteristic, however, is the effect on $\alpha_{I}(M)$, which increases rapidly by a factor of two, reaching a maximum at $M \simeq 1.5 M_{\odot}$, and then falls monotonously. This specific behavior is to some extent due to a rapid drop in $I$ for $M \gtrsim 1.4 M_{\odot}$.

\footnotetext{
${ }^{1}$ Let us remind that non-rotating cold neutron stars $M_{\min } \approx 0.1 M_{\odot}$ (Haensel et al. 2002).
} 


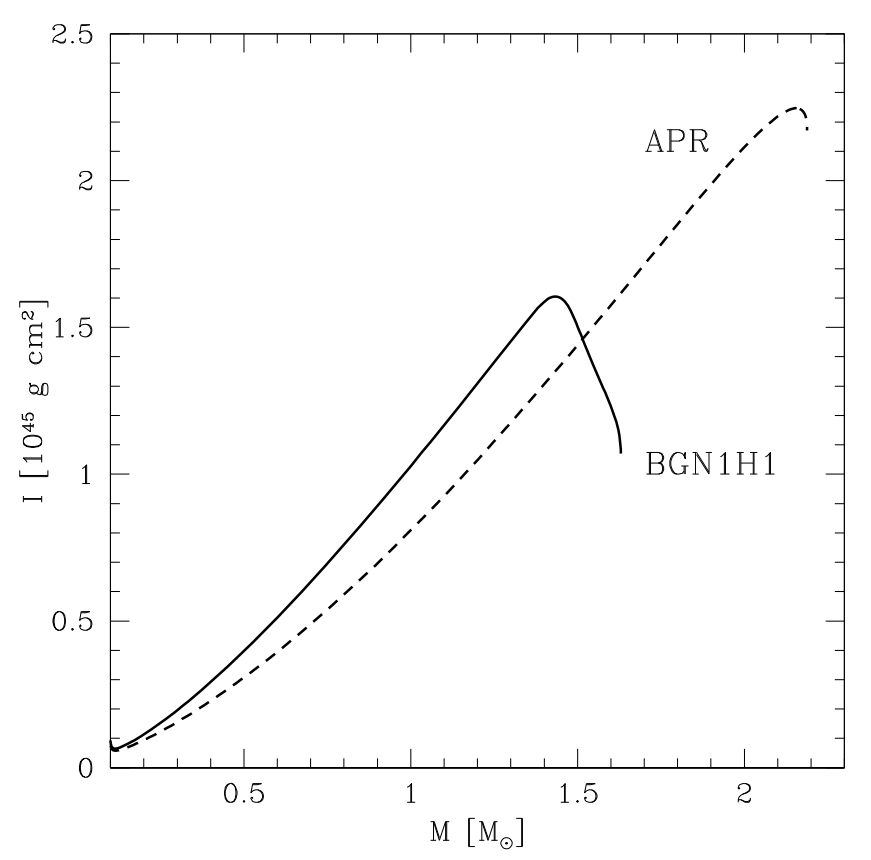

Fig. 3. Moment of inertia for slow, rigid rotation, $I=I_{0}$, versus stellar mass, $M$, for the APR and BGN1H1 EOS1 (Table 1).

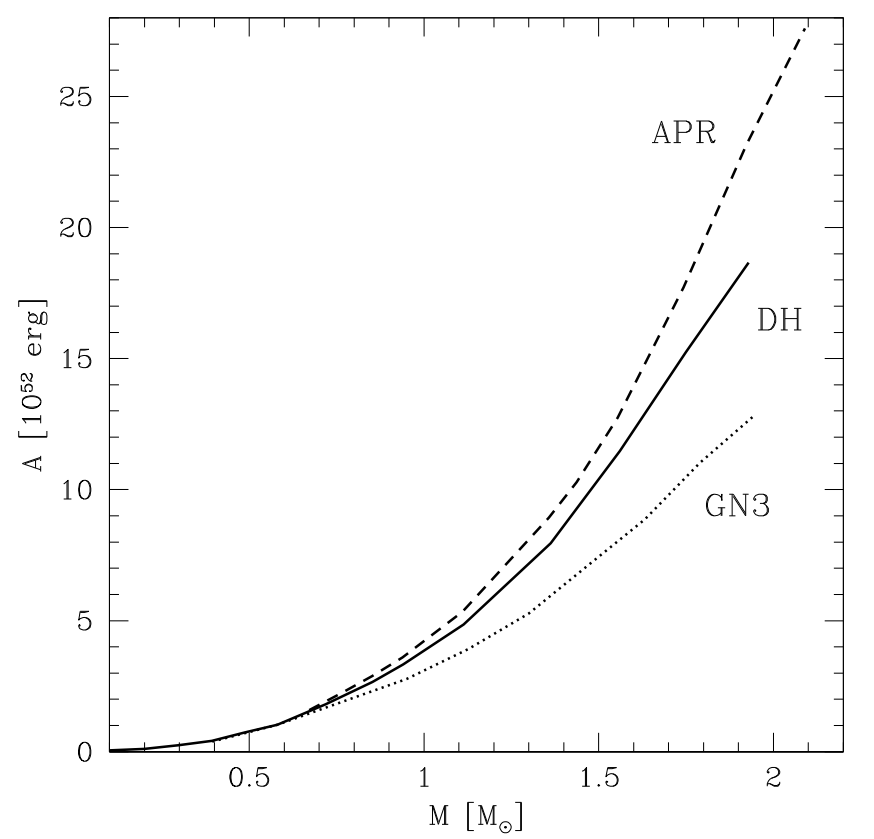

Fig. 4. Parameter $A$ versus stellar mass $M$ for the APR, DH, and GN3 EOSs (Table 1). For more explanations see the text.

\subsection{Energy and oblateness - coefficient $A$}

Using Eqs. (7), (8), and (11) we obtain final expression for $A$ in terms of three $\alpha$-s:

$A=\alpha_{\mathrm{d}}\left(\alpha_{\mathrm{s}} / \alpha_{I}\right)^{2}$

Plots of $A(M)$ for seven realistic EOSs from Table 1 are shown in Figs. 4 and 5. As already explained in Sect. 3.1, high precision 2D calculations were only possible within a limited range of stellar mass, $0.2 M_{\odot} \lesssim M \lesssim 0.9 M_{\max }$. Notice that EOSs in Fig. 4

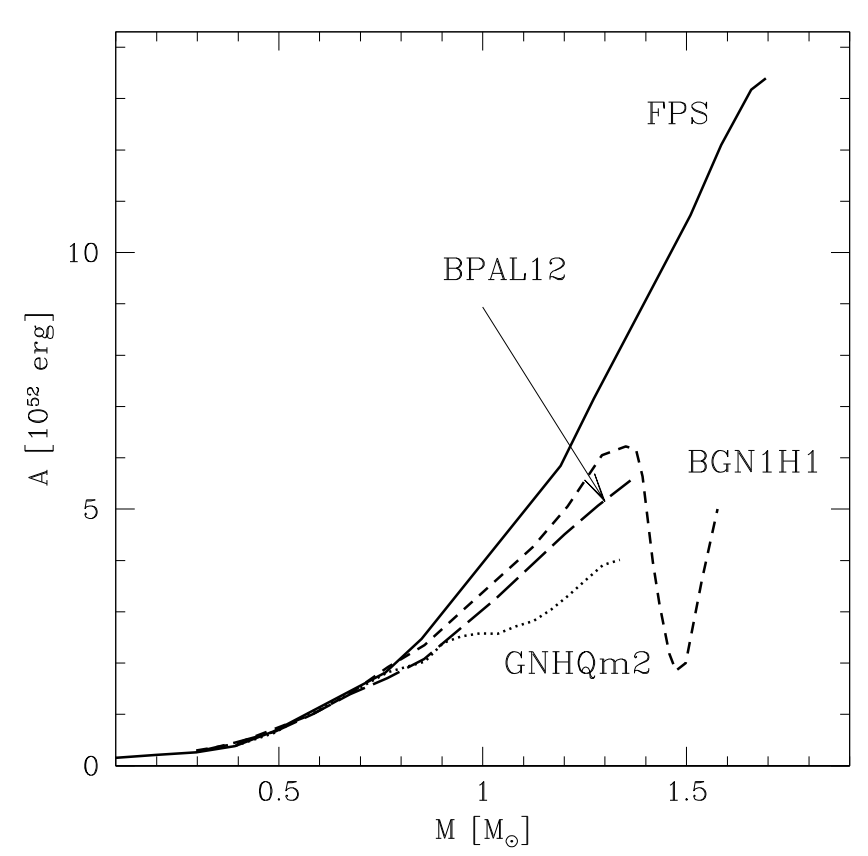

Fig. 5. Same as Fig. 4 but for the BPAL12, FPS, BGN1H1, and GNHQm2 EOSs (Table 1). For more explanations see the text.

have no high-density softening. They lead to monotonously increasing $A(M)$, with a maximum scatter at given $M$ characterized by a factor of two.

In Fig. 5 we compared $A(M)$ for two EOSs with high-density softening, due to hyperonization $(\mathrm{BGN} 1 \mathrm{H} 1)$ and to a transition to a mixed hadron-quark phase (GNHQm2), with two EOSs without such a softening. Hyperon softening implies a dramatic drop (by a factor of five!) for $M \gtrsim 1.4 M_{\odot}$. The appearance of a mixed-phase core leads to quasi-constancy of $A$ for BNHQm2 EOS near $M \simeq 1 M_{\odot}$.

Up to now, $A(M)$ has been calculated using the model of incompressible-fluid stars in the Newtonian theory of gravitation (Baym \& Pines 1971; Pandharipande et al. 1976; Carlini \& Treves 1989; Jones \& Andersson 2001). For such a model, the formula for $A(M)$ was originally derived by Love (1920),

$A=\frac{3}{25} \frac{G M^{2}}{R}$.

As we see in Fig. 6, this formula badly underestimates $A(M)$ for realistic neutron stars with a high-density softening, and it is unable to reproduce a strong drop, due to a high-density softening of the EOS.

For a sufficiently low stellar mass, one can describe the $A(M)$ relations by a universal formula, because the influence of the dense-matter EOS becomes less and less important with decreasing $M$. Consequently, the $A(M)$ relations for different EOSs converge to a single one. An approximate formula (fitted in the $0.1-0.7 M_{\odot}$ range) reads

$A(M)=a_{1}\left(M / M_{\odot}\right)^{b_{1}} 10^{53} \mathrm{erg}$

with $a_{1}=3.87$ and $b_{1}=7 / 3$. The fitting formula works very well, with accuracy of the order of one per cent, for masses less than $0.7 M_{\odot}$. Its quality worsens for higher masses when the $A(M)$ relations become EOS-dependent. Similarly, one can approximate the numbers given by the Newtonian Love's expression, where the true values of $M$ and $R$ are used, by a universal 


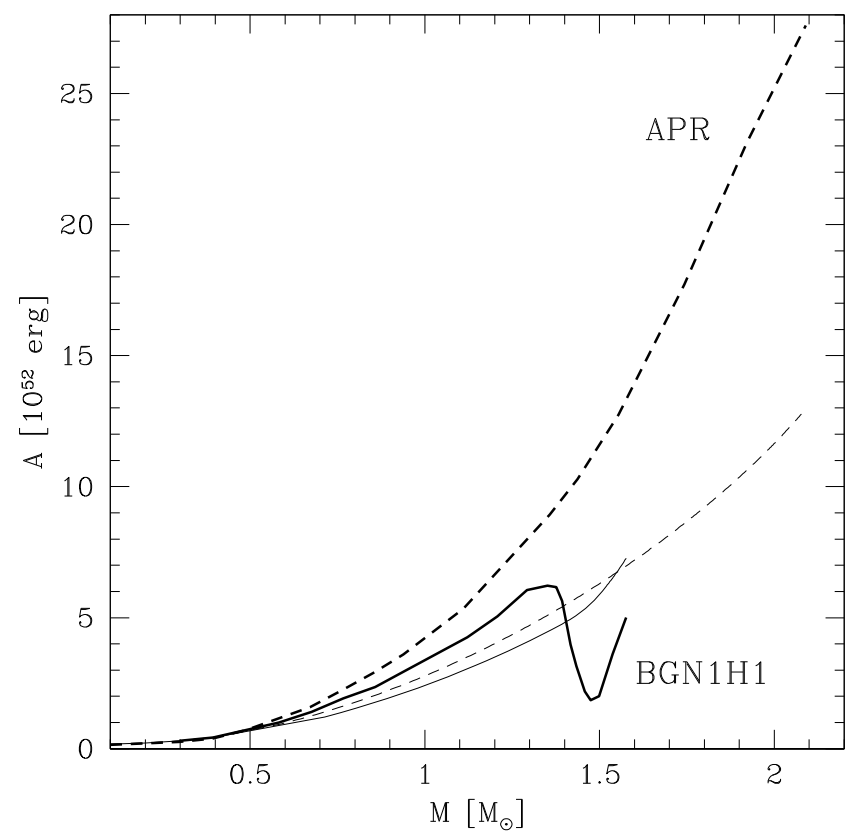

Fig. 6. The coefficient $A$ versus stellar mass, $M$, for the APR (thick dash line) and BGN1H1 (thick solid line) EOSs (Table 1). Thin lines (dash APR, solid - BGN1H1) correspond to a standard incompressible-fluid approximation, Eq. (13).

function $A_{\text {Love }}(M)$ analogous to Eq. (14), but with $a_{1}=2.68$ and $b_{1}=2$. Such a formula for $A_{\text {Love }}(M)$ is quite precise (within a few percent) for masses lower than $0.7 M_{\odot}$.

\section{Shear modulus of the crust}

We follow the notations of Haensel et al. (2007). Deformation of the crust with respect to the equilibrium (ground state) configuration of ions (nuclei) in the lattice implies displacement of ions into new positions $\boldsymbol{r}^{\prime}=\boldsymbol{r}+\boldsymbol{u}$, where $\boldsymbol{u}=\boldsymbol{u}(\boldsymbol{r})$ is the displacement vector (field). In the macroscopic (continuum) limit, $\boldsymbol{r}$ and $\boldsymbol{u}$ are treated as continuous fields. Nonvanishing $\boldsymbol{u}$ induces deformation energy density $\mathcal{E}_{\text {def }}=\mathcal{E}(\boldsymbol{u})-\mathcal{E}_{0}$, where $\mathcal{E}_{0}$ corresponds to the non-deformed (equilibrium) crust. Non-zero $\boldsymbol{u}$ induces forces that tend to return a matter element to the state of minimum energy density $\mathcal{E}_{0}$. These forces result from the elastic strain. The deformation energy is determined by the strain tensor,

$u_{i k}=u_{k i}=\frac{1}{2}\left(\frac{\partial u_{i}}{\partial x_{k}}+\frac{\partial u_{k}}{\partial x_{i}}\right)$,

and the elastic coefficients of the crust. For an ideal bcc lattice, there are two independent elastic shear moduli $b_{11}=\frac{1}{2}\left(c_{11}-c_{12}\right)$ and $c_{44}$ (see, e.g., Kittel 1986). For a classical Coulomb lattice, they were first calculated by Fuchs (1936, see also Ogata et al. 1990): $b_{11}=0.0245 n_{N}(Z e)^{2} / r_{\mathrm{c}}, c_{44}=0.1827 n_{N}(Z e)^{2} / r_{\mathrm{c}}$. Crustal matter is likely to be an isotropic bcc polycrystal, with a single effective shear modulus $\mu$. The shear strain contribution to the stress tensor is then

$\sigma_{i j}^{\mathrm{sh}}=2 \mu\left(u_{i j}-\frac{1}{3} \delta_{i j} \sum_{k} u_{k k}\right)$.

A standard choice in the preceding studies of elastic effects in the neutron star structure and dynamics was $\mu=c_{44}$
(Baym \& Pines 1971; Pandharipande et al. 1976; Mc Dermott et al. 1988, and references therein). This choice significantly overestimates $\mu$. A correct calculation, involving averaging over rotations of Cartesian axes, was done by Ogata et al. (1990), and gives

$\mu=\frac{1}{5}\left(2 b_{11}+3 c_{44}\right)=0.1199 \frac{n_{N}(Z e)^{2}}{r_{\mathrm{c}}}$.

We rewrite this formula in a form suitable for calculations:

$\mu=7.816 \times 10^{28}\left(\frac{\rho_{13}}{A_{\mathrm{c}, 3}}\right)^{4 / 3}\left(\frac{Z}{40}\right)^{2} \mathrm{erg} \mathrm{cm}^{-3}$

where $\rho_{13} \equiv \rho / 10^{13} \mathrm{~g} \mathrm{~cm}^{-3}, A_{\mathrm{c}}$ is the total number of nucleons in the ion cell, $A_{\mathrm{c}, 3} \equiv A_{\mathrm{c}} / 10^{3}$, and $Z$ is the number of protons in an nucleus. This is the formula used in the present paper. It should be stressed that this formula, strictly speaking, holds for the outer crust, where the approximation of point-like nuclei is valid. However, for simplicity we also use Eq. (18) in the inner crust, neglecting the effect of finite nuclear size and possible existence of non-spherical "pasta nuclei" (see the end fragment of Sect. 4.1).

\subsection{Ground-state crust}

This is a standard approximation assumed in the calculations of the neutron-star structure (see Haensel et al. 2007, and references therein). At each pressure, matter is assumed to realize the minimum of the enthalpy per nucleon. This is equivalent to the minimum of the energy per nucleon at every density; however, in contrast to the pressure that is continuous and strictly monotonous in the stellar interior, the density can suffer jumps.

The ground-state approximation may be good for isolated neutron stars formed in core-collapse supernovae. Initial temperature is there $\gtrsim 10^{10} \mathrm{~K}$ and the matter is in nuclear equilibrium. In a few months, following neutron star birth, the temperature falls to $10^{9} \mathrm{~K}$, so that layers of $\rho \gtrsim 10^{8} \mathrm{~g} \mathrm{~cm}^{-3}$ are strongly degenerate. It is tacitly assumed that the matter cools, keeping nuclear equilibrium. As a result, the cooled crust reaches its ground state. Its structure and the EOS can be calculated assuming $T=0$.

In the inner crust, at densities approaching $10^{14} \mathrm{~g} \mathrm{~cm}^{-3}$, the nuclear radius becomes comparable to the distance between nuclei. The effective nuclear charge becomes smaller than $Z$ due to electrons present in nuclear volume, and moreover the nuclei can lose their spherical shape, being replaced by "nuclear pasta" (for a review, see Haensel et al. 2007). However, the very existence of a "pasta nuclei" layer near the bottom of the crust is model-dependent (Haensel et al. 2007). The pasta layers correspond to various phases of liquid crystals and Eq. (18) is not valid for them. Elastic moduli of some pasta phases were calculated by (Pethick \& Potekhin 1998, for a review, see Haensel et al. 2007).

In the present paper, we limit ourselves to the crust models that do not exhibit pasta phases. We mainly use the crust model of Douchin \& Haensel (2001), in which spherical nuclei exist down to the bottom edge of the crust, so that Eq. (18) can be used as a reasonable approximation. In the upper panel of Fig. 7, we show plots of effective shear modulus $\mu$ versus density $\rho$ for two models of the ground-state crust (Douchin \& Haensel 2001; Negele \& Vautherin 1973, the latter one for the inner crust only). Differences between two models are small. 


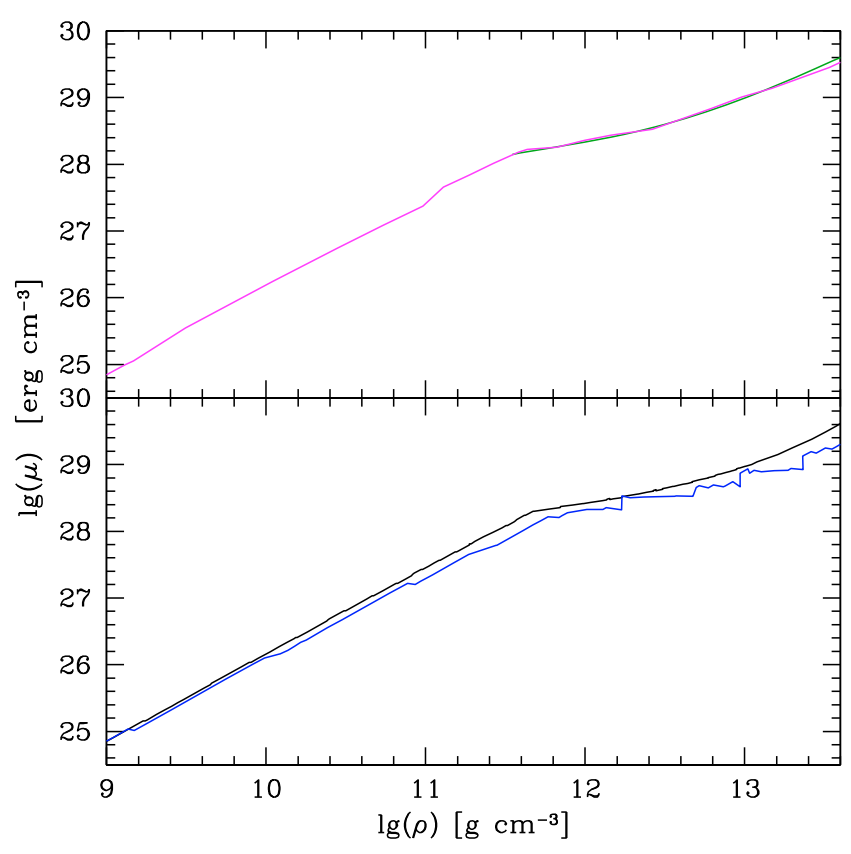

Fig. 7. (Color online) Effective shear modulus $\mu$, Eq. (17), versus $\rho$, for different models of the crust. Upper panel: ground-state crust. Magenta line - Douchin \& Haensel (2001); green line - Negele \& Vautherin (1973), only inner crust. Discontinuities in $\rho$ have been smoothed via interpolation. Lower panel: blue line - an accreted crust model including pycnonuclear fusions (Haensel \& Zdunik 2008). The ashes of X-ray bursts consist of pure ${ }^{56} \mathrm{Fe}$, the crust structure given in Table A.3 of Appendix A of (Haensel \& Zdunik 2008). Black line - ground-state crust, calculated using the same compressible liquid drop model of nuclei as the accreted crust.

\subsection{Accreted crust}

Neutron stars that have gone through a long stage of accretion in the low-mass X-ray binaries have very different crusts from the ground-state one. Accretion could last for as long as $\sim 10^{9}$ years, with typical accretion rates $10^{-10}-10^{-9} M_{\odot} \mathrm{y}^{-1}$. After $\sim 10^{7} /\left(\dot{M} / 10^{-9} M_{\odot} \mathrm{y}^{-1}\right)$ years, the original crust, formed at neutron star birth, is replaced by an accreted one. During formation of an accreted crust, temperature in the stellar interior with $\rho \gtrsim 10^{8} \mathrm{~g} \mathrm{~cm}^{-3}$ is at most a few times $10^{8} \mathrm{~K}$ (Miralda et al. 1990), and the only reactions in compressed matter are those not associated with overcoming the nuclear Coulomb barriers. Before the neutron drip point at $\rho \approx 6 \times 10^{11} \mathrm{~g} \mathrm{~cm}^{-3}$, evolution is mediated by the electron captures. At higher density, neutron emission and absorption also play important rôle. Finally, at $\rho \sim 10^{12}-10^{13} \mathrm{~g} \mathrm{~cm}^{-3}$, the charge of proton clusters becomes so low that it has been speculated that their zero-point vibrations around the crustal lattice sites could possibly allow for the pycnonuclear fusion (Sato 1979).

Accreted crust, produced in the scenario described above, has composition dramatically different from the ground-state one. In particular, nuclei in an accreted crust have much smaller $A$ and $Z$ (Sato 1979; Haensel \& Zdunik 1990a, 2003). Therefore, an accreted crust is softer to an elastic deformation than is the ground-state one. To correctly calculate the difference between shear moduli of the ground state and of an accreted crust, one has to use the same nuclear model. We assume the Mackie-Baym model of nuclei in dense matter (Mackie \& Baym 1977), in the version used in Haensel \& Zdunik (1990a,b, 2003).
Our results are presented in the lower panel of Fig. 7. At a given $\rho$, nuclei in an accreted crust have lower values of $Z$ and $A$ than those in the ground-state crust. The values of $\mu(\rho)$ are correspondingly lower. One notices sharp drops and jumps of $\mu$ of accreted crust. The drops are due to electron captures leading to $Z \longrightarrow Z-2$. In the inner crust, electron capture triggers emission of neutrons, so that both $Z$ and $A$ drop at the threshold density. At some densities above $10^{12} \mathrm{~g} \mathrm{~cm}^{-3}, Z$ may be low enough to allow for pycnonuclear fusion, resulting in a significant jump in $Z$ and $A$, corresponding to a very large jump in $\mu$. However, one has to stress uncertainties in the description of zero point motion of proton clusters carrying with them bound neutrons, in the dense superfluid medium of unbound neutrons. Therefore, this part of the scenario of the formation of accreted crust is plagued by huge uncertainties, and even doubts, about whether the pycnonuclear fusion occurs at all. In contrast to the accreted crust, we get a smooth $\mu(\rho)$ for the ground-state crust, calculated using the same compressible liquid drop model of nuclei. Notice that our model has no shell correction term.

\section{Crust structure: an approximation}

In the simplest approximation, we consider a spherical neutron star built of an ideal fluid. The hydrostatic equilibrium is then determined by the Tolman-Oppenheimer-Volkoff (TOV) equations (see, e.g., Shapiro \& Teukolsky 1983)

$$
\begin{aligned}
& \frac{\mathrm{d} P}{\mathrm{~d} r}=-\frac{G\left(\rho+P / c^{2}\right)\left(m+4 \pi r^{3} P / c^{2}\right)}{r^{2}\left(1-2 G m / r c^{2}\right)}, \\
& \frac{\mathrm{d} m}{\mathrm{~d} r}=4 \pi r^{2} \rho,
\end{aligned}
$$

where $r$ is the circumferential radius and $m(r)$ the (gravitational) mass contained within the sphere of radius $r$. The boundary conditions are $m(0)=0$ and $P(R)=0$, where $R$ is the star radius. The star mass is $M=m(R)$. The bottom of the crust is at $r=R_{\mathrm{b}}$, so that the mass of the crust is $M_{\mathrm{cr}}=M-m\left(R_{\mathrm{b}}\right)$. The pressure at the bottom of the crust is $P_{\mathrm{b}}=P\left(R_{\mathrm{b}}\right)$.

Within the crust, $0<P<P_{\mathrm{b}}, R>r>R_{\mathrm{b}}$ and $M-M_{\mathrm{cr}}<$ $m<M$. We limit ourselves to neutron stars with $M_{\mathrm{cr}} \ll M$, so that $m \approx M$ and $4 \pi r^{3} P / m c^{2} \ll 1$ within the crust. Moreover, within the crust $P / c^{2} \ll \rho$. Under these conditions, Eq. (19) can be rewritten as

$$
\frac{\mathrm{d} P}{\mathrm{~d} r}=-\frac{G M \rho}{r^{2}\left(1-2 G M / r c^{2}\right)}
$$

or, equivalently, as

$\frac{\mathrm{d} P}{\rho}=-G M \frac{\mathrm{d} r}{r^{2}\left(1-2 G M / r c^{2}\right)}$.

Within the crust, i.e., for $0<P<P_{\mathrm{b}}$, we define a dimensionless function of pressure

$\chi(P)=\int_{0}^{P} \frac{\mathrm{d} P^{\prime}}{\rho\left(P^{\prime}\right) c^{2}}$.

Notice that $\chi(P)$ is determined solely by the EOS of the crust. Using Eq. (22), one obtains $\chi$ versus $r$,

$\chi=\frac{1}{2} \ln \left[\frac{1-r_{\mathrm{g}} / R}{1-r_{\mathrm{g}} / r}\right]$ 
where $r_{\mathrm{g}} \equiv 2 G M / c^{2}$. Defining $a=1-r_{\mathrm{g}} / R$, we obtain $r$ within the crust as a function of $\chi$,

$r=r_{\mathrm{g}} /\left(1-a \mathrm{e}^{-2 \chi}\right)$.

We can now derive a useful formula for an integral of any function $f$ over the crust volume. A proper volume of a spherical shell between $r$ and $r+\mathrm{d} r$ is

$\mathrm{d} V=4 \pi r^{2} \frac{\mathrm{d} r}{\left(1-r_{\mathrm{g}} / r\right)^{1 / 2}}$.

Using Eq. (25) we obtain

$\int_{R_{\mathrm{b}}}^{R} f \mathrm{~d} V=8 \pi R^{3}(1-a)^{3} a^{1 / 2} \int_{\eta_{\mathrm{b}}}^{1} f \frac{\mathrm{d} \eta}{\left(1-a \eta^{2}\right)^{4}}$,

where $\eta=\mathrm{e}^{-\chi}$ and $\eta_{\mathrm{b}}=\eta\left(P_{\mathrm{b}}\right)$. Therefore, for a given function $f(P)$ and for a given value of $P_{\mathrm{b}}$, it is sufficient to calculate a function

$\mathcal{Z}_{f}(a)=(1-a)^{3} a^{1 / 2} \int_{\eta_{\mathrm{b}}}^{1} f \frac{\mathrm{d} \eta}{\left(1-a \eta^{2}\right)^{4}}$

and the value of the integral of $f$ over the crust of any star having the radius and the mass of the star $R$ and $M$ can then be obtained using Eq. (27). In our case $f=\mu$.

\section{Calculation of $B$}

We assume a quadrupolar deformation of the star. Starting from a relaxed rotating configuration $\breve{C}$, characterized by some $\breve{\Omega}$ and $\breve{\epsilon}$, we calculate the hydro-elastic equilibrium configuration $C^{\text {sd }}$ after a continuous, complete spin down to $\Omega=0$. The configuration $C^{\text {sd }}$ is characterized by $\epsilon_{\mathrm{r}}>0$. Within our approximation, the hydro-elastic equilibrium at $\Omega=0$ implies

$b=\frac{\epsilon_{\mathrm{r}}}{\breve{\epsilon}}, \quad B=\frac{b}{1-b} A \approx b A$.

Classical analytic result, valid for a self-gravitating elastic sphere of constant $\rho$ and $\mu$, and volume $V$, is (Love 1920)

$B=\frac{57}{50} V \mu$.

In this case the displacement field $\boldsymbol{u}(\boldsymbol{r})$ is calculated analytically (Love 1920). Baym \& Pines (1971) used this displacement field to calculate $B=B_{\mathrm{BP}}$ for a compressible solid crust (model of Baym et al. 1971b), enveloping a liquid core with EOS of Baym et al. (1971a). Pandharipande et al. (1976) performed calculations of $B$ using two approximations. First, they used the prescription of Baym \& Pines (1971). Second, they calculated $B$ using a modification of Eq. (30) obtained by replacing $57 / 50$ by one, $V$ by crust volume $V_{\mathrm{c}}$, and $\mu$ by the average over the crust volume, $\bar{\mu}$. Therefore, their approximation reads as

$B \approx B_{\text {int }}=\int_{\text {crust }} \mathrm{d}^{3} r \mu(r)$.

Pandharipande et al. (1976) found that $B_{\text {int }}$ is a rather good approximation of $B_{\mathrm{BP}}$.

As shown by Cutler et al. (2003), the use of $\boldsymbol{u}(\boldsymbol{r})$ of constant $\rho$ and $\mu$ solid sphere for a thin solid shell enveloping a fluid core badly overestimates $B$. Using the analytic $\boldsymbol{u}(\boldsymbol{r})$ of Franco et al. (2000) for an incompressible shell, Cutler et al. (2003) derived,

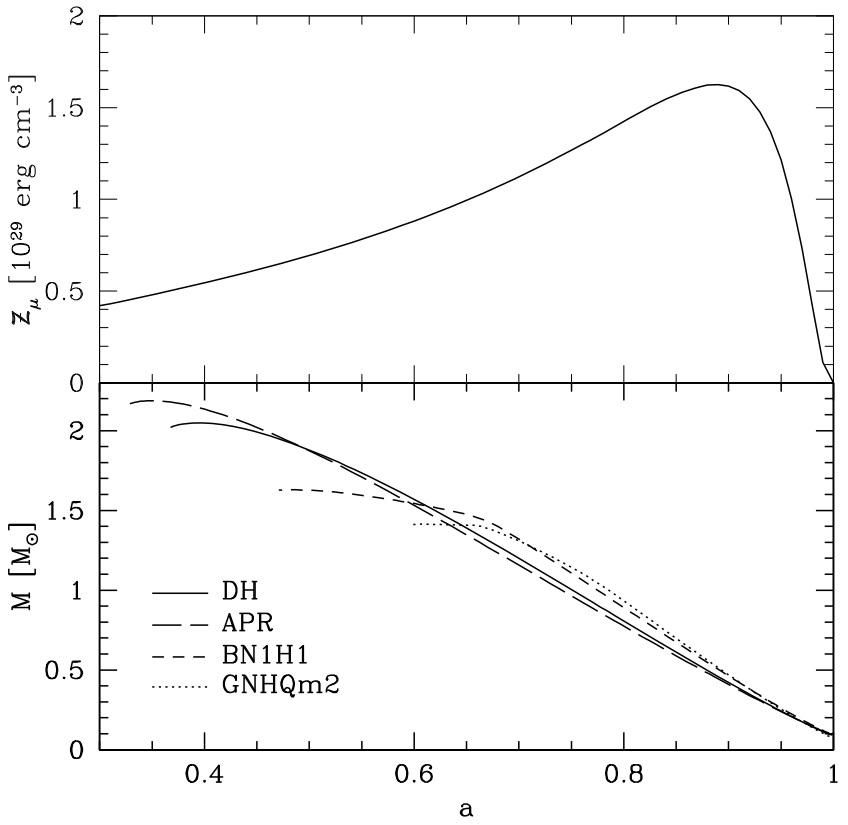

Fig. 8. Upper panel: the function $\mathcal{Z}_{\mu}(a)$, Eq. (28), calculated for the DH model of the ground-state crust, assuming $f(P)=\mu(P)$, Eq. (17). Lower panel: relation between gravitational mass $M$ and $a=1-r_{\mathrm{g}} / R$, calculated for four EOSs of the neutron-star core.

within the Cowling approximation, a correct formula for a thin crust model of constant $\rho$ and $\mu$,

$B_{\text {inc }}=\frac{12}{55} V_{\mathrm{c}} \mu$

The prefactor is smaller by 5.23 than in Eq. (30). However, the use of Eq. (32) with a simple substitution $\mu \longrightarrow \bar{\mu}$ would still badly overestimate the true value of $B$, by a factor $\approx 8$ for a $1.4 M_{\odot}$ star (Cutler et al. 2003). This is due to additional cancellations of different contributions of elastic strain to $\epsilon_{\mathrm{r}}$ (Cutler et al. 2003). The calculations of Cutler et al. (2003) were done using the model of the crust of Negele \& Vautherin (1973), combined with several EOSs of the core. Numerical results, presented in Table 1 of Cutler et al. (2003), were obtained for a $1.4 M_{\odot}$ neutron star.

We do not attempt to find $B$ via solving the hydro-elastic equilibrium equations, like in Cutler et al. (2003). Instead, we approximate $B$ by a generalization of Eq. (31) in the form

$B=0.027 \int_{\text {crust }} \mathrm{d}^{3} r \mu(r)$,

where the prefactor accounts for the real distribution of strain stresses within the crust after the spin down. Assuming a reduction factor $0.027 \approx 1 / 42$ as compared to the Baym-Pines' estimate, we reproduce results of Cutler et al. (2003) quite well.

\section{7. $\Delta \boldsymbol{B}\left(\rho_{\mathrm{b}}\right)$ for the ground-state and accreted crust}

We denote by $\Delta B\left(\rho_{\mathrm{b}}\right)$ the contribution to $B$ of the crust layer with density $\rho_{\mathrm{b}}$ at the bottom. We use the approximation given by Eqs. (33) and (27). Therefore, the value of $\Delta B(\rho)$ is determined by two functions, crust EOS and $\mu(P)$, and by a single, star-structure parameter $a=1-r_{\mathrm{g}} / R$. The dependence of $\Delta B(\rho)$ on the crust model is illustrated in Fig. 9. Three curves were obtained using different crust models. The upper curve was based 


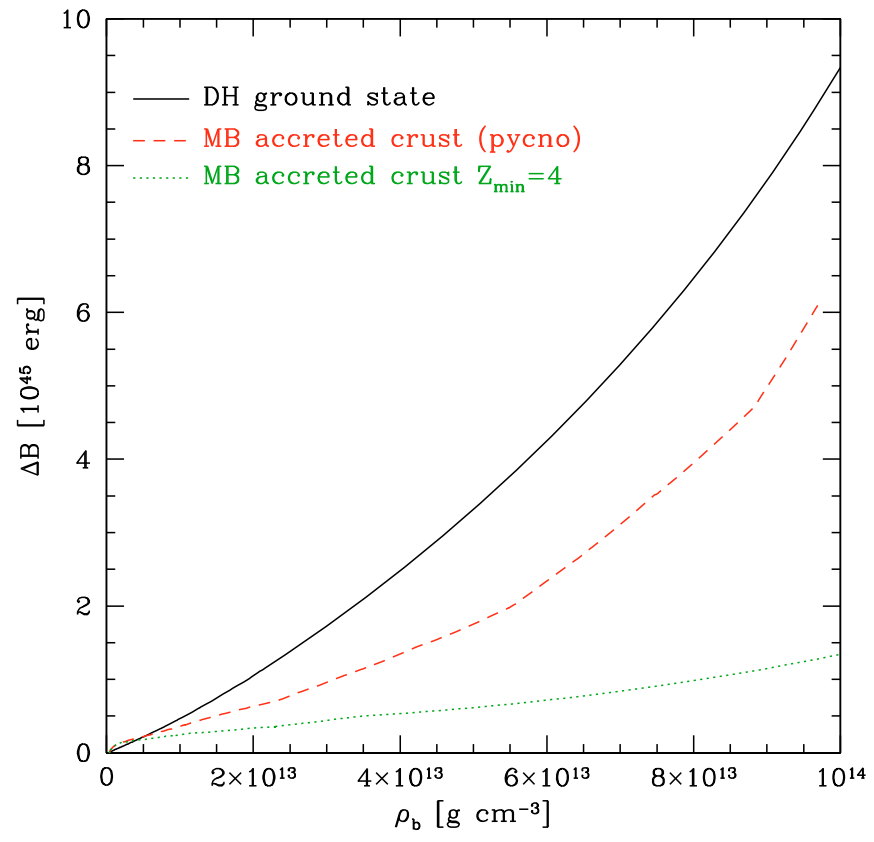

Fig. 9. (Color online) Function $\Delta B\left(\rho_{\mathrm{b}}\right)$ calculated for three models of neutron-star crust and assuming $a=0.65$. This value of $a$ is obtained for a $1.4 M_{\odot}$ star model based on the DH EOS for the crust and core (Douchin \& Haensel 2001). Two MB models differ in the treatment of the pycnonuclear fusion. In both MB models, X-ray ashes are pure ${ }^{56} \mathrm{Fe}$. Green dotted line: artificial blocking of pycnonuclear fusion until $Z_{\min }=4$. Red dashed line: no artificial suppression of pycnonuclear reactions, Table A.3 of Appendix to (Haensel \& Zdunik 2008).

on the ground-state model of Douchin \& Haensel (2001). The intermediate curve was calculated assuming a model of accreted crust from (Haensel \& Zdunik 2008, see caption to Fig. 9). The values of $\mu$ are lower than for the ground-state crust, making $\Delta B\left(\rho_{\mathrm{b}}\right)$ lower, too.

Pycnonuclear reaction rates at $\rho>10^{12} \mathrm{~g} \mathrm{~cm}^{-3}$ are the most uncertain element of an accreted crust model. An uncertainty factor of $\sim 10^{6}$ is common for theoretical calculations of reaction rates (Yakovlev et al. 2006). To visualize the rôle of pycnonuclear fusion in determining $\Delta B$, we (artificially) suppressed these reactions for $Z>Z_{\min }=4$. Such modification has no significant effect on the total deep crustal heating in accreting neutron star (Haensel \& Zdunik 2008). However, as seen in Fig. 9, it has a strong effect on $\Delta B\left(\rho_{\mathrm{b}}\right)$, decreasing it by a factor of two at $2 \times 10^{13} \mathrm{~g} \mathrm{~cm}^{-3}$ and by a factor of five at $10^{14} \mathrm{~g} \mathrm{~cm}^{-3}$. By nearly doubling $Z$ and decreasing the number of nuclei by two in each reaction shell, pycnonuclear reactions have a very strong impact on the crustal rigidity.

\section{Scaling of $B$ with $M$ and $R$}

Jones (2000) derived, within Newtonian gravity, a simple scaling of $\int_{\text {crust }} \mu \mathrm{d} V$ with $M$ and $R$. The starting point was an estimate of the crust thickness $\Delta R$, in the limit of $\Delta R / R \ll 1$. By balancing gravity and pressure at the bottom of the crust, one gets

$\Delta R \propto R^{2} / M$.

In the same approximation, the crust volume scales

$\Delta V=4 \pi R^{2} \Delta R \propto R^{4} / M$.

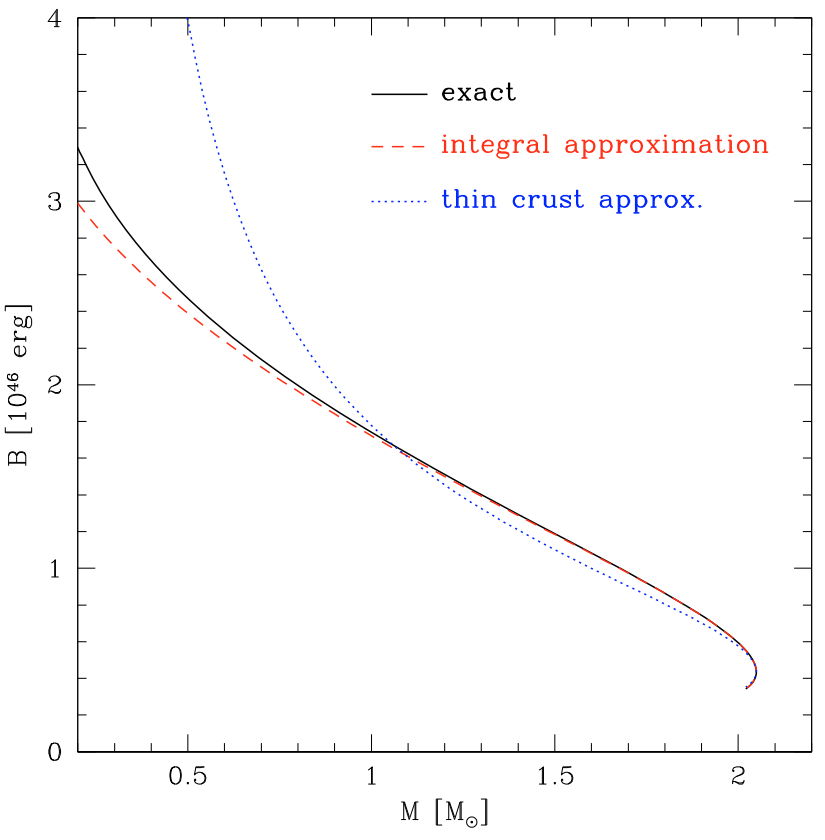

Fig. 10. (Color online) Parameter $B$ versus stellar mass $M$ calculated for the DH EOS (Table 1). Solid line: exact result obtained using Eq. (33). Dashed line: approximation explained in Sect. 5. Thin dotted line: scaling with $M$ and $R$, as derived by Jones (2000), normalized here to the exact value of $B$ at $M=M_{\max }$.

Defining a mean value of the shear modulus, averaged over the crust volume, $\langle\mu\rangle_{\text {crust }}$, one gets (Jones 2000)

$B_{\mathrm{tc}} \propto \Delta V\langle\mu\rangle_{\text {crust }} \propto R^{4} / M$

where the index "tc" recalls that the formula is obtained in the limit of a thin crust. In what follows, we "normalize" the prefactor in the formula for $B_{\mathrm{tc}}$ by requiring that the formula reproduces (for a fixed EOS for the core and the crust) the value obtained by an exact integration $\int_{\text {crust }} \mu \mathrm{d} V$ (see the end of this section).

In Sect. 5 we derive an approximate, but as we will show very precise, formula for $\int_{\text {crust }} \mu \mathrm{d} V$. In particular, we found that for a given $\mu(P)$ and EOS of the crust, the relevant integral over the crust scales in a well-defined manner with $R$ and $M$, the latter scaling entering through the dependence on $a=1-r_{\mathrm{g}} / R$. If we change $M, R \longrightarrow M^{\prime}, R^{\prime}$, we get, in our approximation

$B \longrightarrow B^{\prime}=\left(R^{\prime} / R\right)^{3} \mathcal{Z}_{\mu}\left(a^{\prime}\right) / \mathcal{Z}_{\mu}(a)$

where $\mathcal{Z}_{\mu}(a)$ is given by Eq. (28).

To be specific, let us consider the DH EOS of the crust and the core, for which we also calculated $\mu=\mu(P)$. We fix the prefactor in $B_{\mathrm{tc}}$ by requiring $B_{\mathrm{tc}}=B$ at $M=M_{\max }$, where the crust is the thinnest. In Fig. 10 we compare $B(M)$ obtained using three approximations. The method based on Eq. (27) is very precise, even for $M \approx 0.3 M_{\odot}$ when the crust is no longer thin, while for $M>0.5 M_{\odot}$ its precision is remarkably high. Approximation $B_{\mathrm{tc}}$ is reasonably good only for $M>M_{\odot}$ and provided the value of $\langle\mu\rangle_{\text {crust }}$ is properly chosen, which requires a "normalization" by an exact value of the integral at a selected value of $M$. For $M<M_{\odot}, B_{\text {tc }}$ diverges badly from the precise $B(M)$, and the scaling given by Eq. (36) cannot be used. 

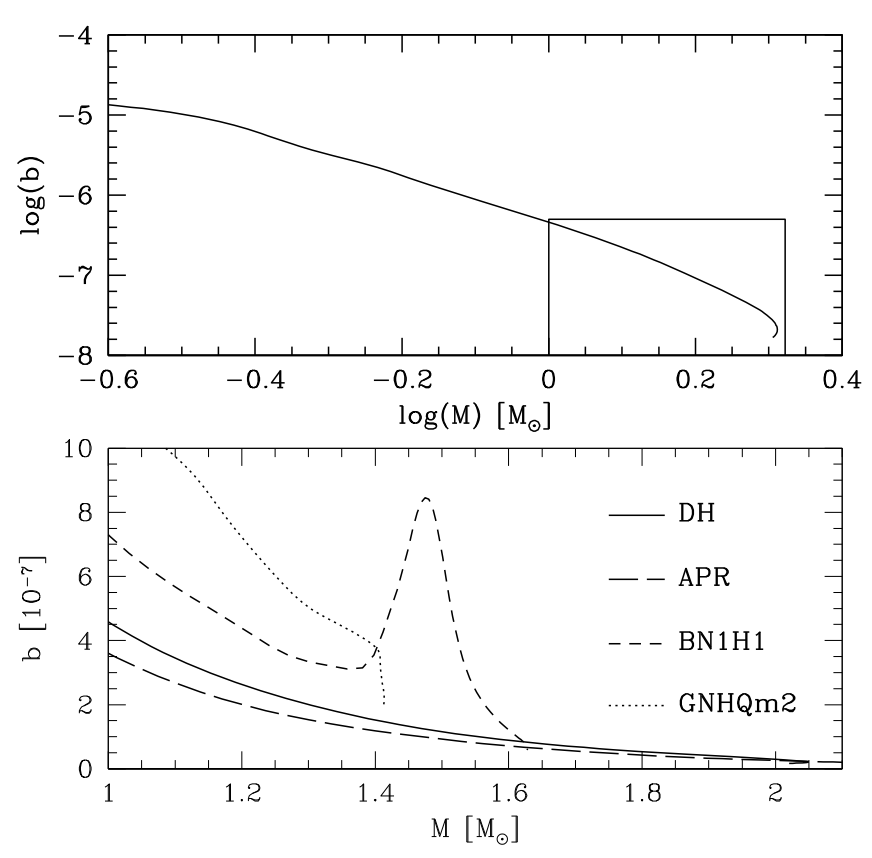

Fig. 11. Crustal rigidity $b$ versus stellar mass. Upper panel: $\log -\log$ plot, which shows results for the DH model of the ground-state crust and core for $0.2 M_{\odot}<M<M_{\max }$. Lower panel: zoomed segment of $b(M)$ for $M>1 M_{\odot}$ (within a rectangle in the lower-right corner of the upper panel) for the DH model of the ground-state crust combined with several EOSs of the core.

\section{Numerical results for crustal rigidity $b$}

Our results are presented in Fig. 11. In the upper panel we show our results for $b(M)$ calculated using the DH model of the crust and core, for $0.25 M_{\odot}<M<M_{\max }(\mathrm{DH})=2.05 M_{\odot}$. In the lower panel we have plotted $b(M)$ for $M>1 M_{\odot}$, calculated using the DH model of the crust and four different EOSs of the core.

\subsection{Nucleon cores}

As examples, we used the DH and APR EOSs for the core. The core EOS does not exhibit any strong softening due to hyperonization or a phase transition, so $b(M)$ is monotonous. It increases with decreasing $M$ because of a monotonous decrease in $A$ (Fig. 4) and an increase in $B$ due to an monotonous increase in the crust volume. Crustal rigidity is a sensitive function of $M$; the value of $b$ at $M=1.0 M_{\odot}$ is ten times greater than at $M=2 M_{\odot}$. For a "standard" mass $1.4 M_{\odot}$ we get $b \approx 2 \times 10^{-7}$, which is within the range of $b=1.5-4.4 \times 10^{-7}$, obtained by Cutler et al. (2003) for the FPS crust (Lorenz et al. 1993) and Negele-Vautherin crust (Negele \& Vautherin 1973), each combined with two different EOSs of the core (see Table 1 of Cutler et al. 2003).

The increase in $b$ with decreasing $M$ is so strong that $b\left(0.3 M_{\odot}\right) \approx 50 b\left(1.4 M_{\odot}\right)$ and we were forced to use the logarithmic scale to plot $b$ in the whole considered mass range. Even at $M=0.3 M_{\odot}$, the maximum elastic strain effect on neutron star structure, measured by $b \sim 10^{-5}$, is a very small perturbation of the perfect-fluid model. However, one also has to notice that the applicability of the small deformation approximation to neutron stars with $M<1 M_{\odot}$, rotating at a few hundred $\mathrm{Hz}$, can be questioned.
Consider, for example, $f=641 \mathrm{~Hz}$, corresponding to PSR B1937+26. For illustration, we use properties of the lowmass neutron stars calculated for the DH EOS of the crust and core by Haensel et al. (2002). At $f=641 \mathrm{~Hz}$, the equatorial radius of a $0.6 M_{\odot}$ star is $17.8 \mathrm{~km}$, to be compared with $12.6 \mathrm{~km}$ for a nonrotating star of the same mass. The polar flattening is characterized by $r_{\text {pole }} / r_{\text {eq }} \approx 0.6$ (here $r$ is the radial coordinate). The equatorial thickness of the crust is four times that at the poles. Actually, at $f=641 \mathrm{~Hz}$, stars with $M<0.6 M_{\odot}$ cannot exist in hydrostatic equilibrium: they are destroyed due to the mass shedding from the equator.

If DH EOS of the core is replaced by the APR one, the change in $b(M)$ is small. Namely, $b(M)$ is somewhat lower, due to a higher stiffness of the APR EOS.

\subsection{Hyperon cores}

A strong softening of the core EOS due to hyperonization has a dramatic effect on the high- $M$ segment of $b(M)$. Namely, the crustal rigidity is no longer monotonous, and after reaching a rather narrow maximum of nearly $10^{-6}$ at $\sim 1.5 M_{\odot}$, it falls monotonously, down to about $10^{-7}$, at $M=M_{\max }(\mathrm{BGN} 1 \mathrm{H} 1)=$ $1.65 M_{\odot}$. This behavior is easily understood in terms of a specific dependence of $A$ on $M$ (see Fig. 5), characterized by a deep and narrow minimum around $\sim 1.5 M_{\odot}$. A minimum in $A(M)$ is reflected by a maximum in $b(M)$.

\subsection{Exotic cores}

Exotic cores are represented by the GNHQm2 EOS, with a phase transition to quark matter via a mixed quark-hadron state. The softening effect implies a low value of $M_{\max }$, high compactness of "hybrid stars" and rapid shrinking of the crusts with $M$ approaching $M_{\max }$. This results in a rapid drop in $b$ near $M_{\max }$. The effect of a softening due to mixed state is seen in Fig. 5. It is represented by flattening of the $A(M)$ curve GNHQm2, and it is more gradual than a violent softening by a hyperonization, as seen in curve BGN1H1 in the same figure.

\section{Discussion and conclusion}

In the present paper we have studied the dependence of the $A$ and $B$ parameters of the Baym-Pines model of the hydro-elastic equilibrium of rotating neutron stars on the stellar mass and on the EOS. We considered seven different EOSs of the liquid core and several models of neutron star crust.

Parameter A. It was calculated using high-precision 2D simulations in General Relativity, so it can be considered as exact, in contrast to the previous estimates based on the incompressible fluid model. We developed a systematic procedure for calculating $A(M)$. This procedure is based on linear expansions of global parameters of rotating neutron stars of a fixed baryon mass in the square of rotation frequency as measured by an observer at infinity. Three different coefficients of the quadratic term measure, respectively, the anisotropy of the inertia tensor $\left(\alpha_{I}\right)$, oblateness of shape $\left(\alpha_{\mathrm{s}}\right)$, and increase in the energy as measured in the star's rest frame $\left(\alpha_{\mathrm{d}}\right)$. Combined, they give $A=\alpha_{\mathrm{d}}\left(\alpha_{\mathrm{s}} / \alpha_{I}\right)^{2}$. A precise calculation of $A(M)$ was possible for $0.2 M_{\odot} \lesssim M \lesssim 0.9 M_{\max }$. Our exact values of $A(M)$ are usually much higher than those given by the standard formula based on the incompressible fluid model, used in the previous calculations. For $M<0.7 M_{\odot}$ the values of $A(M)$ are determined by a low-density segment of the EOS (similar for all EOSs) and are 
given by a universal formula of precision on the order of one percent. For $M>0.7 M_{\odot}$, values of $A(M)$ are EOS dependent and diverge with increasing $M$. For EOSs without a high-density softening (only nucleons and leptons), the values $A(M)$ increase monotonously with $M$. For EOSs with a high-density softening (due to hyperons or a phase transition), $A(M)$ can have a strongly non-monotonous high-mass segment, where $A$ can drop strongly with an increasing $M$. For example, softening by hyperons in the BGN1H1 EOS makes $A$ drop by a factor of about three for $M$ increasing from $1.4 M_{\odot}$ to $1.5 M_{\odot}$. Generally, the EOS dependence of $A(M)$ for $M \approx 1.5 M_{\odot}$ is very strong, and $A$ can increase by a factor of five when going from hyperon-softened EOS to a stiff nucleonic EOS of the APR type.

Crustal rigidity. We derived an approximate formula for the integral of the elastic strain over the crust volume, whose validity goes beyond a thin crust approximation. Our formula can also be used for low-mass neutron stars. We considered three different models of the crust, two for the ground-state crust and one for an accreted crust. The values of $B$ for different models of the ground-state crust and the same core EOS show little model dependence. In contrast, the accreted crust is significantly less rigid (lower $B$ ) than the ground-state one. Results obtained for $M=1.4 M_{\odot}$ with the ground-state $\mathrm{DH}$ crust and nucleonic cores are within the range of values obtained by Cutler et al. (2003); however, we found a strong dependence of $b$ on the star's mass. In the range $1.0 M_{\odot}-M_{\max }$, parameter $b$ varies by an order of magnitude, and one notices a characteristic dependence of relation $b(M)$ on the EOS of the core. For nucleonic cores, $b(M)$ is monotonic. However, hyperonization may lead to a strong maximum in $b(M)$, associated with soft hyperon cores and a deep minimum in $A(M)$. A phase transition via a mixed quark-hadron state implies a strong drop in $b$.

Pycnonuclear reactions and crustal rigidity. We have shown that pycnonuclear reactions, if they proceed, play a crucial rôle for rigidity of accreted crust, because every time they occur they nearly double $Z$. However, theoretical pycnonuclear reaction rates are notorious for their huge uncertainties. There is also a basic difficulty in describing of aggregates of neutrons, bound to the proton clusters, bound in turn in the nuclear potential well created by neutrons, as "nuclei" immersed in a superfluid of dripped (unbound) neutrons. The effect of dripped neutrons on the zero-point vibrations of such "nuclei" and on the fusion rate still needs to be calculated. We use a compressible liquid drop model of nuclei in the inner crust. Assuming that pycnonuclear fusions are suppressed for $Z>Z_{\min }=4$, we get $B$, which is five times smaller than for a model without such an artificial suppression. This may be contrasted with deep crustal heating in accreting neutron stars, where the total heat release per one accreted nucleon was found to be insensitive to the suppression of pycnonuclear reactions (Haensel \& Zdunik 2008).

This paper is first in a series devoted to the interplay of rotation and shear strain in neutron stars. The forthcoming papers will deal with hydro-elastic equilibrium of rotating neutron stars, including the case of hypothetical solid neutron-star cores, predicted by some theories of dense matter. Elastic strain can support non-axial mass distributions of rotating neutron stars, therefore becoming sources of continuous, strictly periodic gravitational waves. Such models of gravitational wave sources are of particular interest in the expected era of gravitationalwave astronomy, which could be (hopefully) opened by LIGO, VIRGO, and other gravitational-wave observatories in the coming decade.

Acknowledgements. This work was partially supported by the Polish MNiSW grant No. N20300632/0450, and by the LEA Astrophysics Poland-France (Astro-PF) program. M.B. was also supported by the Marie Curie Fellowships no. MEIF-CT-2005-023644 and ERG-2007-224793.

\section{References}

Akmal, A., Pandharipande, V. R., \& Ravenhall, D. G. 1998, Phys. Rev. C, 58, 1804

Baym, G., \& Pines, D. 1971, Ann. Phys., 66, 816

Baym, G., Bethe, H. A., \& Pethick, C. J. 1971a, Nucl. Phys. A, 175, 221

Baym, G., Pethick, C., \& Sutherland, P. 1971b, ApJ, 170, 299

Balberg, S., \& Gal, A. 1997, Nucl. Phys. A., 625, 435

Bombaci, I. 1995, in Perspectives on Theoretical Nuclear Physics, ed. I. Bombaci, A. Bonaccorso, A. Fabrocini, et al. (Pisa: Edizioni ETS), 223

Carlini, A., \& Treves, A. 1989, A\&A, 215, 283

Carter, B., \& Quintana, H. 1975, Ann. Phys., 95, 74

Cutler, C., Ushomirsky, G., \& Link, B. 2003, ApJ, 588, 975

Douchin, F., \& Haensel, P. 2001, A\&A, 380, 151

Franco, L. M., \& Link, B., \& Epstein, R. I. 2000, ApJ, 543, 987

Friedman, J. L., Ipser, J. R., \& Parker, L. 1986, ApJ, 304, 115

Fuchs, K. 1936, Proc. Roy. Soc. London A, 153, 622

Glendenning, N. K. 1985, ApJ, 293, 470

Glendenning, N. K. 2000, Compact Stars: Nuclear Physics, Particle Physics, and General Relativity (New York: Springer)

Haensel, P., \& Zdunik, J. L. 1990a, A\&A, 227, 431

Haensel, P., \& Zdunik, J. L. 1990b, A\&A, 229, 117

Haensel, P., \& Zdunik, J. L. 2003, A\&A, 404, L33

Haensel, P., \& Zdunik, J. L. 2008, A\&A, 480, 459

Haensel, P., Zdunik, J. L., \& Douchin, F. 2002, A\&A, 385, 301

Haensel, P., Potekhin, A. Y., \& Yakovlev, D. G. 2007, Neutron Stars 1, Equation of state and structure (New York: Springer)

Jones, D. I. 2000, Ph.D. Thesis, Univ. Wales, Cardiff

Jones, D. I., \& Andersson, N. 2001, MNRAS, 324, 811

Kittel, C. 1986, Introduction to Solid State Physics (New York: Wiley)

Lorenz, C. P., Ravenhall, D. G., \& Pethick, C. J. 1993, Phys. Rev. Lett., 70, 379

Love, A. E. H. 1920, Mathematical Theory of Elasticity (Cambridge: Cambridge Univ. Press)

Mackie, F. D., \& Baym, G. 1977, Nucl. Phys. A, 285, 332

McDermott, P. N., Van Horn, H. M., \& Hansen, C. J. 1988, ApJ, 375, 679

Miralda-Escude, J., Haensel, P., \& Paczynski, B. 1990, ApJ, 362, 572

Munk, W. H., \& McDonald, G. J. F. 1960, The Rotation of the Earth (Cambridge: Cambridge Univ. Press)

Negele, J.W., \& Vautherin, D. 1973, Nucl. Phys. A, 207, 298

Ogata, S., \& Ichimaru, S. 1990, Phys. Rev. A, 42, 4867

Owen, B. J. 2005, Phys. Rev. Lett., 95, 211101

Pandharipande, V. R., Pines, D., \& Smith, R. 1976, ApJ, 208, 550

Pandharipande, V. R., \& Ravenhall, D. G. 1989, in Proc. NATO Advanced Research Workshop on nuclear matter and heavy ion collisions, Les Houches, ed. M. Soyeur, et al. (New York: Plenum), 103

Pines, D., \& Shaham, D. 1972a, Nature Phys. Sci., 235, 43

Pines, D., \& Shaham, D. 1972b, Phys. Earth Planet. Inter., 6, 103

Pethick, C. J., \& Potekhin, A. Y. 1998, Phys. Lett. B, 427, 7

Ruderman, M. 1992, in Structure and Evolution of Neutron Stars, ed. D. Pines, R. Tamagaki, \& S. Tsuruta (Redwood City CA: Addison-Wesley), 353

Sato, K. 1979, Prog. Theor. Phys., 62, 957

Shapiro, S. L., \& Teukolsky, S. A. 1983, Black Holes, White Dwarfs, and Neutron Stars: The Physics of Compact Objects (New York: Wiley)

Yakovlev, D. G., Gasques, L., \& Wiescher, M. 2006, MNRAS, 371, 1322 